\title{
Space: telescopes reveal the way forward
}

\section{Astronauts' skills should be saved for major work in exploring the origins of the Universe.}

Sir - Following the Columbia tragedy, scientists must re-evaluate the role of astronauts in scientific research (see Nature 421, 559; 2003). We should ask astronauts to participate only in frontline research that would otherwise be prohibitively difficult. The continued exploration of the origins of the Universe and life itself with astronomical telescopes is such an area. For example, primitive life on an extrasolar planet could be detected as a chemical transformation of its atmosphere, as happened on Earth a billion or more years ago. But the telescopes must be of unprecedented size and sophistication. To be realistic, they will need continuing development in space, with controls and instruments upgraded as experience is developed.

Such evolution has been crucial to the success of the Hubble Space Telescope. Astronauts have carried out repairs and upgrades, transforming it into a long-lived and exceptionally productive observatory. Some advanced telescopes will build on this experience. The 10-metre X-ray Evolving Universe Spectroscopy (XEUS) telescope will be built at the International Space Station and operated nearby. A 10metre successor to Hubble (see simulation in figure) for the optical and ultraviolet band could be similarly developed, eventually achieving the sensitivity to study extrasolar terrestrial planets.

Large infrared telescopes can also be extremely powerful, but present a special problem, because they need to be cryogenically cooled to be sensitive to faint heat sources. Thus they cannot be operated conveniently in low orbit where they would be warmed by Earth's radiated heat. The first large telescope of this type, the James Webb Space Telescope, is planned for operation a million miles away. But with a 6-metre aperture it will be much bigger than its cryogenic precursors, larger and more complex even than Hubble. If its parts wear out or need upgrading, or if its folded optics do not deploy correctly, it might be possible to bring the telescope back to a rendezvous as close as the Moon (Nature 419, 666; 2002), but at considerable risk to astronauts. Otherwise we must launch an entirely new telescope at the same scale, or abandon the project.

For still larger and more complex cryogenic telescopes and interferometers, strategies to combine remote operation and more convenient astronaut access are clearly desirable. For example, a telescope could be assembled in low-Earth orbit as two linked spacecraft: a large but simple

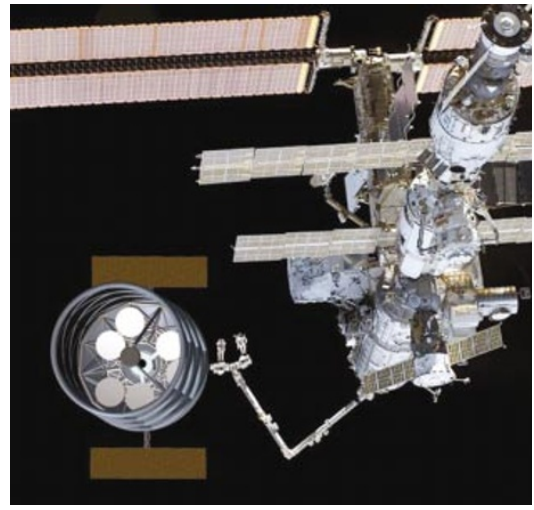

Potential: a 10-metre successor to Hubble.

one housing the main mirrors and built to survive radiation damage; the other much smaller, lighter and containing the sensitive instruments and active optics controls. After verification of performance in orbit at room temperature, the two would be transferred independently to the remote operating point. The large structure would use efficient but slow solar-electric propulsion; the smaller instrument would be moved quickly through the radiation belts by conventional means. Both would carry enough fuel to allow a return to low-Earth orbit for repairs and upgrades by astronauts when necessary. Attachment and detachment could be achieved robotically, as with the unmanned supply vehicles that dock at the space station. If astronauts again travel far from Earth, a manned lunar station would have great scientific potential and be a stepping-stone to a martian exploration. In general, the Moon is not an attractive telescope location, given its huge monthly thermal cycle. But the south lunar pole has attractive features for people and telescopes. Hydrogen is present, probably as water ice, and because the Moon's spin axis is not much tilted there are peaks experiencing uninterrupted sunshine.

A habitation on one of these could have constant solar power and a roomtemperature environment. A telescope here would maintain constant deflection under gravity as it rotated once a month to track the stars. It could be built and tested in the sunshine, and for cryogenic operation would be cooled down simply by erecting a shallow bowl of multilayer insulation to screen out radiation from the Sun and Earth. For servicing or repairs, the bowl would be lowered to warm up the telescope. A 20-metre telescope built in this way would be of quite extraordinary sensitivity and flexibility, and would represent a huge accomplishment for lunar-based astronauts.

Roger Angel

Department of Astronomy and Steward Observatory, University of Arizona, 933 North Cherry Avenue, Tucson, Arizona 85721, USA

\section{Space: next step is an International Moon Base}

Sir - Your far-sighted Editorial (Nature $421,559,2003$ ) on the future of human space exploration in the wake of the Columbia accident is a welcome contrast to some of your more sceptical comments about this subject in the past. Your call for vision, and clear endorsement of the exploratory value of human spaceflight, was a breath of fresh air. I would like to make two points.

First, you identify three possible future roles for humans in space: as geologists exploring near-Earth asteroids; as servicing crews for telescopes at L2; and as explorers on Mars. There are indeed good reasons for believing that all these activities, and more, would benefit from a human presence in space, but we must not forget the Moon.

The Apollo missions pioneered the use of astronauts as field geologists, and I shudder to think what the textbooks would now have to say about the early history of the Solar System had Apollo not taken place. Even today, one can scarcely attend a scientific meeting on the subject without seeing geochemical and isotope analyses of Apollo samples presented in one context or another. Yet Apollo, quite literally, only scratched the surface of the Moon, and there is so much more to learn. Moreover, while Mars does indeed beckon, we should probably learn to operate successfully on the Moon before attempting this much greater challenge.

Second, your Editorial implied that human space exploration is solely NASA's responsibility. But if such exploration is worthwhile, as I believe it is, we should all share in the costs and the risks as well as in the benefits. The pre-eminent value of the much-maligned International Space Station is not so much the science to be performed on it, valuable though that is, but the model it provides for international cooperation in space. We should aim to build on this experience to develop a 
global human spaceflight infrastructure from which science can only benefit an international Moon base being the obvious next step.

Ian Crawford

School of Earth Sciences, Birkbeck College,

Malet Street, London WC1E 7HX, UK

\section{Illustration: database pictures tell a true story}

Sir - Julio M. Ottino in his Commentary "Is a picture worth 1,000 words?" (Nature 421, 474-476; 2003) divides images into two categories: those that convey data and those that illustrate scientific ideas. He defends the practice of image manipulation as sometimes being a necessary part of the process of discovery, yet expresses concern about the blurring of the line between fantasy and reality in scientific illustration.

It may be that the two categories are not that distinct. The European Space Agency's programme Innovative Technologies from Science Fiction for Space Applications assumes that even the most fantastic illustrations may be a useful stimulus to science. On the other hand, images that purport to convey factual data may convey something else entirely. Many observers, for example, were convinced that they could see little human beings in the sperm images produced by the sixteenth-century microscopist Antoni van Leeuwenhoek, and they recorded this observation as fact, presumably influenced by their beliefs.

Today, we can and should capture not just an image but information documenting the process of image creation itself, from the original unmodified data to the final web-ready or journal-ready artwork. If questions arise about the interpretation of an image, we need to be able to go back to the raw data or, if this is impossible, at least to have a full record of what was done to it, and why. For example, in performing video-enhanced contrast microscopy, one always subtracts a digital background 'mottle' image from the live video stream to obtain the mottle-free video frames that are recorded, and viewers need to be informed that background subtraction has been carried out. Similar arguments relate to the point spread functions used in preparing the deconvolved fluorescence images mentioned in the article.

Of course, as in the days of pen-and-ink illustration, scientists should still consider the purpose served by each image in their publications, and should make these objectives clear to their readers. Scientists today have the additional responsibility of recording the processes by which images are created, so that these can be accurately replicated. Currently, such information is usually held only in the laboratory from which the image came, if it is recorded at all.

The BioImage Database Project (http://www.bioimage.org), part of ORIEL (Online Research Information Environment for the Life Sciences; http://www.oriel.org), will be a searchable database of multidimensional images of biological specimens. From its outset, we have felt it essential to acquire not just high-quality source images, but also the various images derived from them, and detailed metadata documenting the process of their creation. We believe that this approach addresses many of the issues raised by Ottino, and should be far more widely adopted.

Chris Catton, David Shotton

Image Bioinformatics Laboratory,

Department of Zoology, University of Oxford,

South Parks Road, Oxford OX1 3PS, UK

\section{Illustration: images fail to portray dynamic skies}

Sir - An example of the visual deception practised by scientific illustrators seeking greater impact - as described by Julio M. Ottino in his Commentary (Nature 421, $474 ; 2003)$ - is the increasingly prevalent practice of adding foreground stars and background galaxies to images generated from numerical simulations of galactic collisions. Examples appear in National Geographic 203, 2, 2003; and on the Gadget website at www.mpa-garching. mpg.de/gadget. Another is one of my simulations, published unadulterated on the cover of Nature on 9 March 1989, which was later "improved" by the imagemakers at NASA's Space Telescope Science Institute (http://hubblesite.org/ newscenter/archive/2001/22/video/c) without my consent.

Although one may agree that superimposed stars and galaxies add a bit of visual interest to the blankness of cyberspace, it is disturbing that these additions are entirely static. In reality, foreground stars hurtle past like snowflakes in a blizzard, and even background galaxies change noticeably over the hundreds of millions of years that are represented in the computer simulations. Static foreground stars and background galaxies undermine the basic idea of a dynamic Universe that these images and animations presumably attempt to convey.

Joshua E. Barnes

Institute for Astronomy, University of Hawaii, Honolulu, Hawaii 96822, USA

\section{Swift publication would reward good reviewers}

Sir - I completely agree with T. Clausen and O. B. Nielsen, who say in Correspondence (Nature 421, 689; 2003) that peer-reviewing needs to be adequately rewarded for the system to work efficiently. However, their proposed remedies, such as mandatory inclusion of reviews in CVs, do not seem promising, not least because this is already routinely done and does not seem to change the general picture.

While toiling on my considerable backlog of manuscripts to review, I came up with a scheme that might stand a better chance of improving the situation: diligent reviewing could be rewarded by speeding up publication of the reviewer's own research.

Currently, most of the prominent journals impose a strict deadline (typically between 7 and 14 days) on reviewers, yet the level of compliance is dismally low, as any author waiting for longer than two months for reviews of a submitted manuscript can testify.

My suggestion is that a researcher who meets the deadline for a particular journal with satisfactory reviews on, say, six consecutive occasions within a two-year period is guaranteed by the journal that his or her next submission will be given privileged status and reviewed within that same deadline. If the journal's editor cannot receive reviews of a privileged submission before the deadline, the journal would have to make a decision based on the other referees' comments or, if none had been received, accept the paper as it stands.

A possible variant is for journals that reject many submissions without review to guarantee to review a privileged submission (again, within the deadline). This is unlikely to burden these highprofile journals with piles of junk, because an individual writing six or more useful reviews for one of them within a limited time span is, by definition, a highly competent researcher.

Of course, a crucial aspect is the notion of a 'useful' review: to earn a privileged submission, reviews would have to be of high quality, as judged by the journal's editors, rather than one-liners. I believe that such direct feedback between a researcher's own publications and reviewing activity could seriously improve the peer-review system.

Feedback loops do wonders in biological systems; they just might work for science, too. Health, Bethesda, Maryland 20894, USA 